\title{
Traumatic vertebral artery dissection presenting with incomplete congruous homonymous quadrantanopia
}

\author{
Albert I Matti ${ }^{1}$, Andrew W Lee² and Celia S Chen*3
}

\begin{abstract}
Background: To describe a rare presentation of vertebral artery dissection (VAD) as a small but congruous incomplete homonymous hemianopia demonstrating use of visual field testing in the diagnosis.

Case presentation: A 30 year old woman had been unwell for 4 months with difficulty focusing, vertigo, dizziness and a feeling of falling to the right. A small but congruous right inferior homonymous quadrantanopia was found on examination leading to further investigation that uncovered a vertebral artery dissection and multiple posterior circulation infarctions including a left occipital stroke matching the field defect.

Conclusions: We describe an atypical case of VAD presenting with a small congruous quadrantanopia. This is a rare but significant condition that predisposes to multiple thromboembolic infarction that may be easily misdiagnosed and a high index of suspicion is required to make the diagnosis.
\end{abstract}

\section{Background}

Vertebral arterial dissection (VAD) may occur after neck trauma [1,2], and along with carotid artery dissections may account for $25-30 \%$ of ischaemic strokes in patients $<50$ years [3]. Diagnosis can be difficult due to its rarity and varied symptoms. Described here-in a case of VAD resulting in multiple cerebral infarctions. The diagnosis was made after finding a small congruous homonymous quadrantanopia.

\section{Case presentation}

A 30 year old woman became unwell after swimming with crocodiles while suspended under water in a protective cage in the Australian Northern Territory. She was vertiginous after coming out of the water and required admission to a tertiary hospital emergency department and was discharged with a diagnosis of vestibular neuronitis.

* Correspondence: Celia.Chen@health.sa.gov.au

${ }^{3}$ Department of Ophthalmology, Flinders Medical Centre and Flinders University, Flinders Drive, Bedford Park, Adelaide SA 5042, Australia

Full list of author information is available at the end of the article
She continued to experience symptoms of vertigo, difficulty focusing, dizziness and a feeling of falling to the right for over 4 months and saw various healthcare providers with no cause found. She had a plain computerized tomography of the head without contrast that was reported as normal. Eventually she was referred to a neuro-ophthalmologist for the complaint of "difficulty focusing" and automated perimetry showed a small incomplete congruous homonymous quadrantanopia (Figure 1). Magnetic resonance imaging (MRI) of the brain revealed a left occipital infarct. There were multiple areas of bilateral posterior circulation infarcts involving the cerebellum, and right thalamus (Figures 2a). Echocardiography, carotid ultrasound and hypercoagulable screens were negative. A computerized tomography (CT) angiogram showed a right vertebral dissection with both thinning and a double lumen of the vertebral artery (Figure $2 \mathrm{~b}$ ). The patient was placed on anticoagulant therapy with no further neurological symptoms.

Vertebral artery dissection (VAD) is a potentially fatal condition where disruption of the vessel wall results in thromboembolism and subsequent ischemic stroke. It may occur following neck injury resulting in neck exten- 


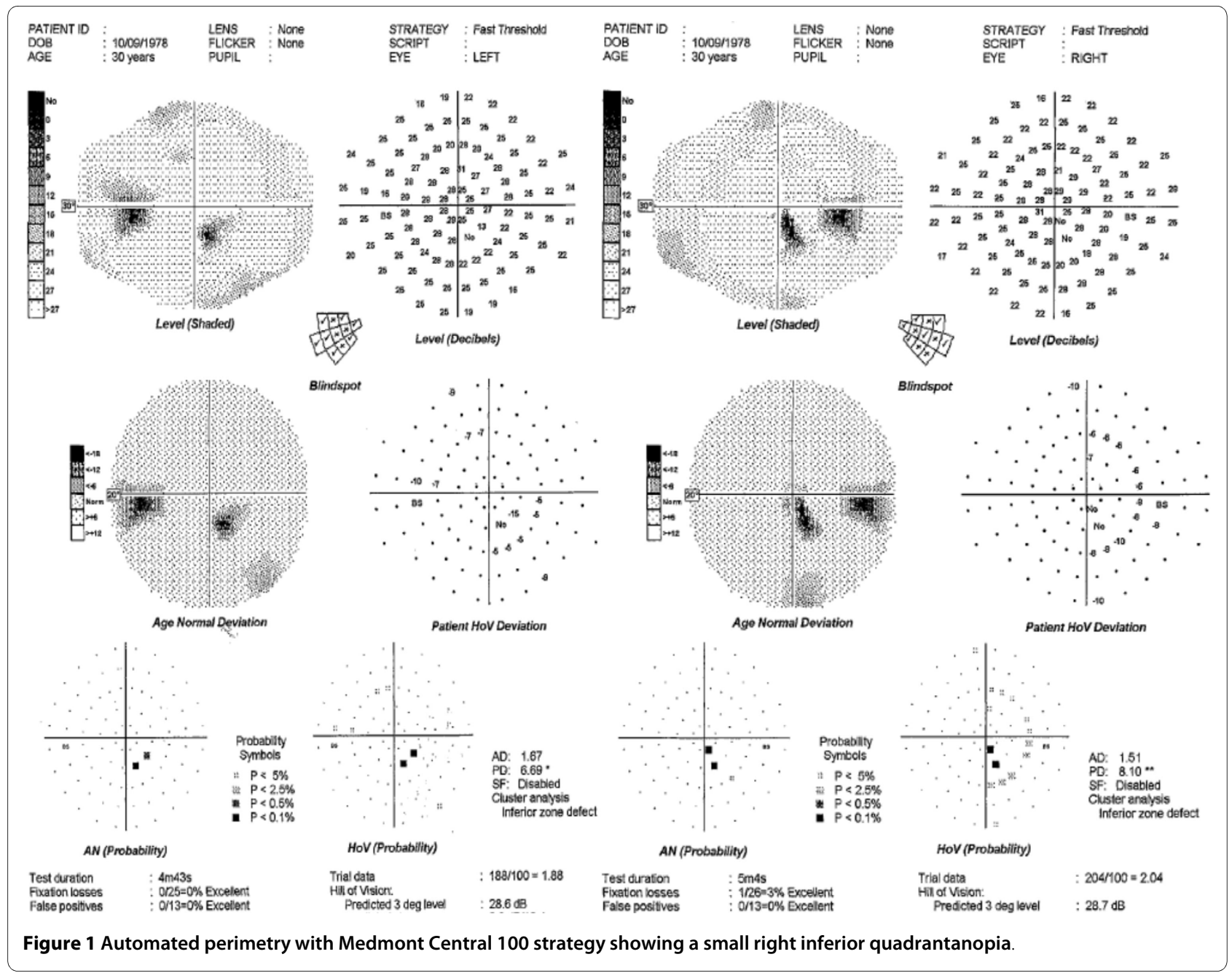

sion, flexion or rotation [1-3]. Symptoms include neck or head pain, partial Horner's syndrome and those of ischemic stroke in its involved territory [3]. The presentation may vary from case to case. The patient likely sustained the VAD following minor neck torsion injury during swimming, followed by a shower of emboli causing multiple posterior circulation infarcts. The congruous nature of the quadrantanopia pointed to an occipital lobe infarct.

This case demonstrates the importance of ancillary imaging in making the diagnosis. $\mathrm{CT}$ angiography provides accurate imaging of the vessel lumen and dissection length. It can identify VAD in both large and medium vessel dissections in the neck [4]. T1-weighted MRI is an alternative but may not detect initial stages of intramural haemorrhage seen in early VAD[1]. It is for this reason that, recent studies suggest that CTA is the preferred imaging modality to identify cervical dissections, especially for VAD [5].
Management of VAD to prevent further embolic stroke is done on a case by case basis. There is no data to support the therapeutic superiority of anticoagulants over antiplatelet agents[6]. In this case the patient had no contraindications to anticoagulants including intracranial arterial dissection, large stroke, enlarging intramural haematoma and/or high risk of bleeding. The presence of multiple cerebral emboli with no contra-indication favoured the use of anticoagulants in this case.

\section{Conclusion}

In conclusion, we describe an atypical case of VAD presenting with a small congruous quadrantanopia. This is a rare but significant condition that may be easily misdiagnosed and a high index of suspicion is required to make the diagnosis.

\section{Consent}

Written informed consent was obtained from the patient for publication of this case report. 


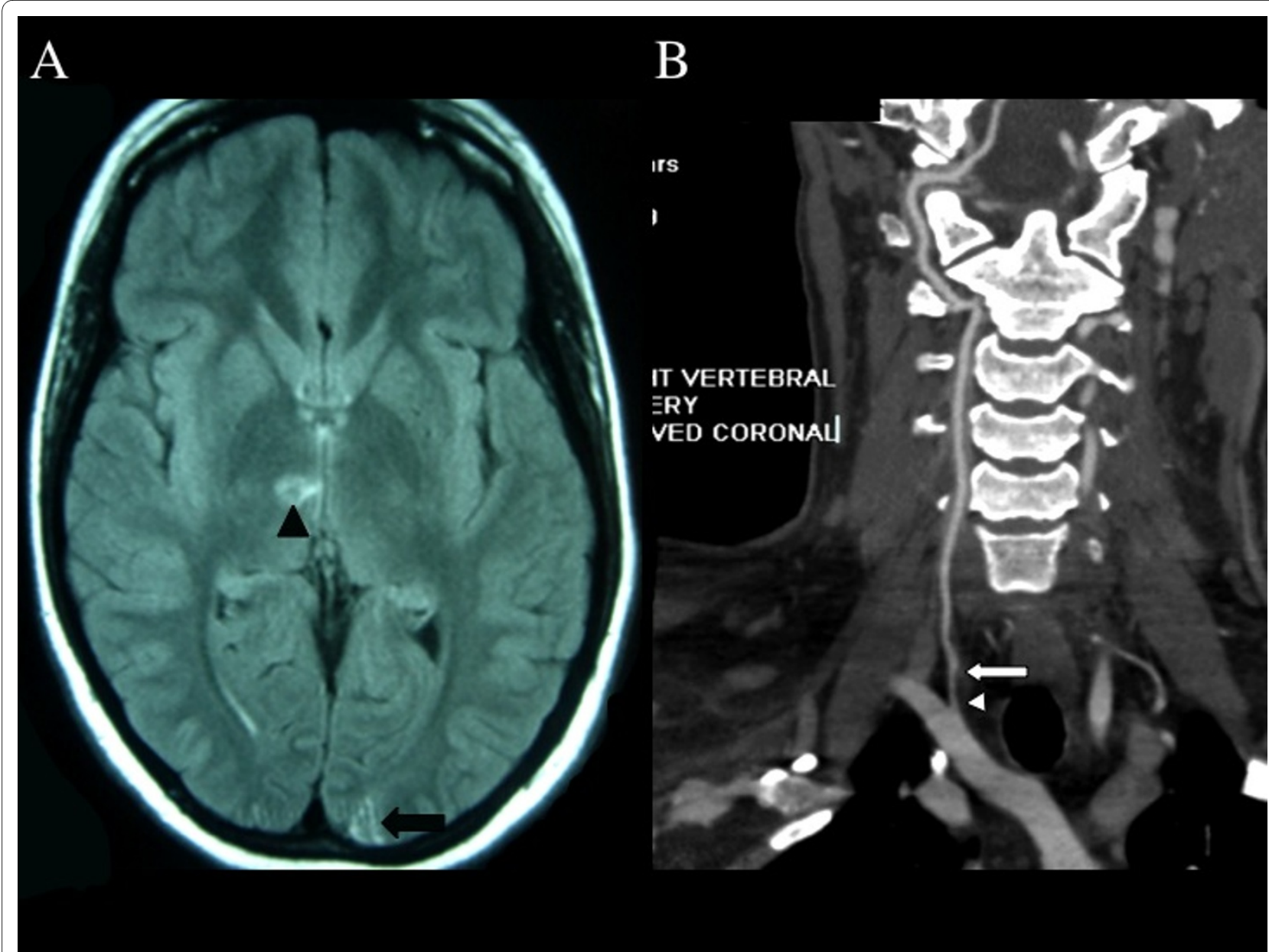

Figure 2 Diagnostic imaging results. (A) Magnetic Resonance Imaging Axial T2 FLAIR section showing a left occipital infarct (arrow) and also a right paramedian thalamic infarct (arrowhead). (B) Coronal view of the right vertebral artery on CT angiogram showing thinning of the artery (arrowhead) and shadowing of the dissected section of the vertebral artery (arrows).

\section{Competing interests}

The authors declare that they have no competing interests.

\section{Authors' contributions}

AM carried out preparation and drafted the manuscript. CSC and AWL were the treating doctors involved in the investigation and management of the case. All authors read and approved the final manuscript.

\section{Acknowledgements}

There were no funding sponsorship associated with this manuscript. Drs. CSC and AWL are supported by the Australian National Health and Medical Research Council National Institute of Clinical Studies Fellowship.

\section{Author Details}

'Department of Ophthalmology, Flinders Medical Centre, Flinders Drive, Bedford Park, Adelaide, SA 5042, Australia, 2Regional director of Stroke

Medicine, Southern Adelaide Health Service, Flinders Medical Centre, Flinders Drive, Bedford Park Adelaide, SA 5042, Australia and ${ }^{3}$ Department of Ophthalmology, Flinders Medical Centre and Flinders University, Flinders Drive, Bedford Park, Adelaide SA 5042, Australia

Received: 15 November 2009 Accepted: 19 May 2010

Published: 19 May 2010

\section{References}

1. Menon RK, Norris JW: Cervical Arterial Dissection - Current Concepts. Ann N Y Acad Sci 2008, 1142:200-217.
2. Ansari SA, Parmar H, Ibrahim M, Gemmete JJ, Ghandi D: Cervical Dissections: Diagnosis, Management, and Endovascular Treatment. Neuroimag Clin N Am 2009, 19:257-270

3. Kim YK, Schulman S: Cervical artery dissection: Pathology, epidemiology and management. Thromb Res 2009, 123:810-821.

4. Villablanca JP, Rodriguez FJ, Stockman T, Dahliwal S, Omura M, Hazany S, Sayre J: MDCT angiography for detection and quantification of small intracranial arteries: comparison with conventional catheter angiography. AJR Am J Roentgenol 2007, 188:593-602.

5. Vertinsky AT, Schwartz NE, Fischbein NJ, et al.: Comparison of multidetector $\mathrm{CT}$ angiography and MR imaging of cervical artery dissection. AJNR Am J Neuroradiol 2008, 29:1753-60.

6. Menon R, Kerry S, Norris JW, Markus HS: Treatment of cervical artery dissection: a systematic review and meta-analysis. I Neurol Neurosurg Psychiatry 2008, 79:1122-7.

\section{Pre-publication history}

The pre-publication history for this paper can be accessed here: http://www.biomedcentral.com/1471-2415/10/14/prepub

doi: 10.1186/1471-2415-10-14

Cite this article as: Matti et al., Traumatic vertebral artery dissection presenting with incomplete congruous homonymous quadrantanopia BMC Ophthalmology 2010, 10:14 